\title{
MECHANICAL AND ELECTRICAL AUTOMATIC CONTROL SYSTEM BASED ON PLC
}

\author{
Chucong Zhong \\ International Education school, North China Electric Power University, Beijing 102206, China \\ E-mail: zchuncongedu@163.com
}

\begin{abstract}
To optimize the existing automatic control system of paper machine and improve the quality of paper, a mechanical automatic control system of paper machine based on programmable logic controller (PLC) was researched and designed. First, the development status of the paper machine control system was introduced. Then, according to the paper making process, the key points and difficulties of the paper machine control system design was summarized. In view of constant tension control, load distribution control and speed chain synchronization control, the control mechanism and control strategy were analysed respectively. Then, the auto disturbance rejection control (ADRC) algorithm was introduced, and the simulation model of the speed chain synchronization control system was built in the toolbox of MATLAB. Moreover, the ADRC algorithm was applied to the simulation model of the speed chain synchronization control. The simulation results showed that the speed chain control system based on ADRC had better performance than traditional PID control in steady state performance, dynamic performance and disturbance rejection performance. It is concluded that the speed chain synchronization control system with active disturbance rejection controller is better than the ordinary PI controller in regulating time, overshoot and disturbance resistance.
\end{abstract}

Keywords: PLC; mechanical and electrical; automatic control; tension control

\section{Introduction}

The process of paper making is complex and involves a very wide range of knowledge. Therefore, the paper making industry is a kind of complex process industry [1]. Nowadays, industrial countries in the world are moving toward the direction of large-scale, intelligent and integrated development.

To improve the quality of paper production, reduce production costs, enhance the core competitiveness of enterprises, save energy and reduce emissions, the paper industry is also constantly improving its level of automation [2]. The process of paper industry is very complex, and this feature determines the difficulty of paper machine control. In the early stages of development, it was in the simple control phase of applying routine regulation meters. Nowadays, system operation uses a control system based on a robust and reliable advanced controller such as PLC. The level of automation in the paper industry's control system is rapidly growing at an unprecedented scale and speed. In developed industrial countries such as Europe and the United States, the high-speed paper machine has been widely promoted and used [3]
Under normal circumstances, the paper production system consists of the following components: paper machine process control system (PCS), paper machine quality control system (QCS), paper machine drive control system, paper machine web monitoring system (WMS), paper machine web inspection system and machine monitoring system (MMS) Of course, because of the difference between the type and size of the paper machine, not all paper production systems must include all seven of these systems [4]. Of the seven systems described above, the paper machine QCS, paper machine PCS, machine control system (MCS) and paper machine drive control system are standard configuration of ordinary paper machine [5].

The paper making machine automatic control system studied in this paper mainly refers to the paper machine drive control system in the above seven systems. In this research, paper making machine automatic control system is taken as the background, and the key control problems are analysed and simulated. Based on the actual situation of a factory, a PLC-based paper making machine automatic control system is designed. 


\section{Theoretical Analysis}

\subsection{Basic requirements for the automatic control system of paper machine}

The electric drive control system of different paper machine is different because the variety, production and production mode of the paper are different. There are also many types of paper making machines based on wet paper process. Because the same wet process is used, then the approximate process should be the same. Through the analysis and research of its technological process, some basic requirements for the paper machine automatic control system can be summed up as follows [6].

The working speed of a paper machine can be adjusted in a larger range. Nowadays, the paper machine is developing in a diversified direction.

A paper machine may need to be able to produce many kinds of paper, and different kinds of paper are different for the speed of work. This requires that the working speed of a paper machine can be adjusted in a larger range when the production variety is replaced [7]. The speed ratio of the general paper machine is 1 : 2 to $1: 6$. For paper making machines with less change in production, the general speed regulation ratio is $1: 2$, such as newsprint machine. The speed ratio of the printing paper and the writing paper machine is generally 1: 4 . However, for industrial technology paper and advanced paper making machine with more variety of production, the speed regulation ratio needs to reach 1:6. The speed adjustment ratio of individual paper machine needs to reach $1: 10$. When the speed ratio reaches $1: 10$, it can basically adapt to the production of various kinds of paper [8].

Due to the strict requirements for paper tension between the parts, tension control is also necessary so as not to cause any production accidents such as paper fold or paper breakage due to the imbalance of tension. Besides, for the convenience of field operation and management control system, the system should have certain instrument display function, such as displaying some speed, voltage, current value and so on.

In addition, the system also displays some fault signals to facilitate the troubleshooting [9].

The paper machine control system should also have a better communication interface, and can realize all kinds of communication functions. From the contents of the previous paper making process and control system, it is known that the papermaking machine automatic control system is only a subsystem of the whole paper making machine control system.
Since it is a subsystem, it is necessary to communicate with other systems to exchange data, such as data. It is also required that the automatic control system of paper machine should have a complete communication interface to achieve communication between the parallel system and the upper and lower level systems [10].

\subsection{Mechanism analysis and control strategy of speed chain control}

According to the requirements of the wet paper making process, the pulp will first be transformed into paper by the mesh. Then, through the press section, the paper is elongated through the various processes of press section. After entering the drying cadre through the pressing section, the function of the drying cadre is mainly to dry the moisture contained in the paper. Here, the paper will shrink after it is dried [11]. In the whole process, to ensure the normal production of paper making, for the wet part, it mainly finishes the web tension. The paper of this part is wet, so it is generally required that there is a gradual increase in the speed of the part in the rear than in the front part. For cadres, it mainly finishes the drying process, and the web of this part will gradually dry with the drying step by step. Therefore, it is generally required that the latter part has a gradually reduced speed than the previous ones [12]. Through the above process analysis, it is concluded that every part of the control system of paper machine is running at different speeds. Of course, this speed is not irregular. It can also be said that the speed of each branch is a proportional relation, and it is a speed synchronization system [13].

The transmission control system of a paper machine includes many transmission groups. At least one drive motor in each drive unit needs to take part in the synchronous speed control of the whole system. Finally, the motor of the whole system is coordinated with each other to realize the speed chain synchronization control. However, the speed chain synchronization control scheme we have to discuss is what kind of method can be used to achieve the mutual coordination between the motors. According to the synchronous way, there are two main modes of synchronous control of multi motor speed: the mode of mechanical synchronization and the mode of synchronous electric control. With the rapid development of electronic technology, the mode of mechanical synchronization has been used less now. However, the synchronous control strategy under the synchronous mode of electrical control mainly includes parallel synchronization control, master slave synchronization control and cross coupling synchronization control. 


\section{Methodology}

\subsection{Actual process introduction and control system structure of a paper industry}

The automatic control system is designed for a long network multi cylinder paper machine in a paper industry, and the mode of speed regulation is selected for AC frequency conversion. The paper making process of the paper machine is divided into eight parts: net part, vacuum, main pressure, first drying, large cylinder, second drying, pressing and coiling. The specific process is shown in figure 1.

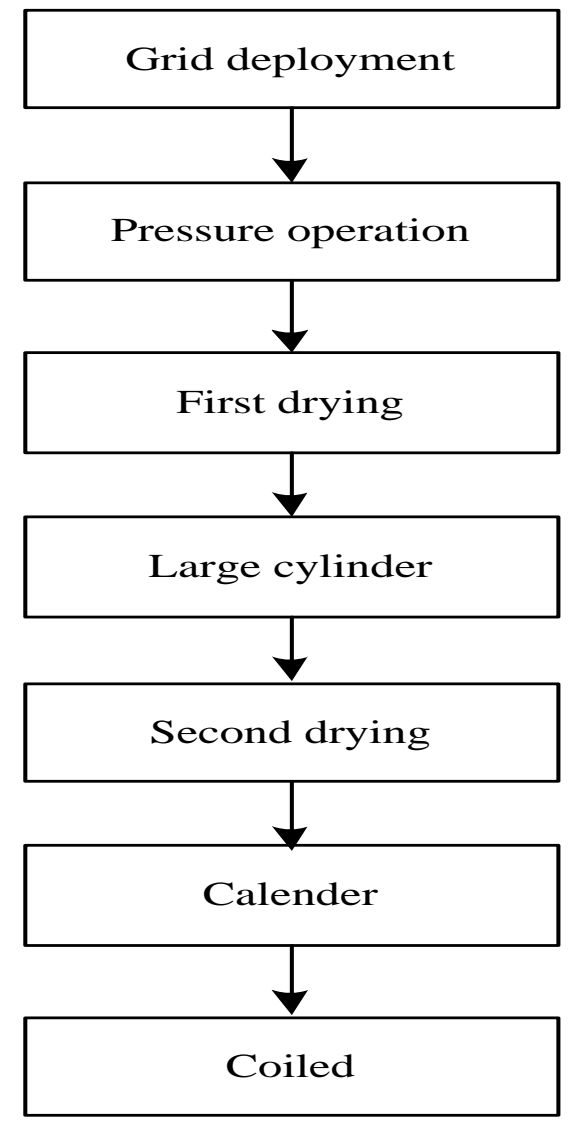

Fig. 1 The producing process of a paper industry
The whole drive control system consists of 8 main drive points and several auxiliary drive points. The specific parameters of the main drive point are shown in table 1.

Table 1 List of main drive point

\begin{tabular}{|c|c|c|}
\hline Number & Name & Power \\
\hline 1 & Net part & $55 \mathrm{KW}$ \\
\hline 2 & Vacuum & $45 \mathrm{KW}$ \\
\hline 3 & Main pressure & $30 \mathrm{KW}$ \\
\hline 4 & First drying & $37 \mathrm{KW}$ \\
\hline 5 & Large cylinder & $22 \mathrm{KW}$ \\
\hline 6 & Second drying & $30 \mathrm{KW}$ \\
\hline 7 & Pressing & $22 \mathrm{KW}$ \\
\hline 8 & Coiling & $22 \mathrm{KW}$ \\
\hline
\end{tabular}

The speed synchronization is a three-phase AC asynchronous motor that is dragged by the eight inverters listed on the table. The speed chain control of these eight drive points is realized by PLC control. The place where the constant tension control is required is between the final coiler and the press.

The role of the coiling section is to roll the web transferred from the calendar into a roll of finished paper. In the process of coiling, the production accidents of paper breaking and paper fold are easy to occur, which affect the quality of paper. The place for load distribution is in the net and the vacuum part. The web from the net also contains a lot of water, which needs to be further dehydrated in the vacuum part. The net and the vacuum are dragged by two AC asynchronous motors. In the production process, the load rate of two motors is same. It avoids the situation that the uneven load may cause the broken paper or felt torn and even cause the motor fault. Finally, the normal production is affected.

Because the environment of the paper making machine industry is poor, and there are many kinds of disturbances. Therefore, the system adopts the PLC with high stability as the main controller. The structure of the control system is shown in figure 2 .

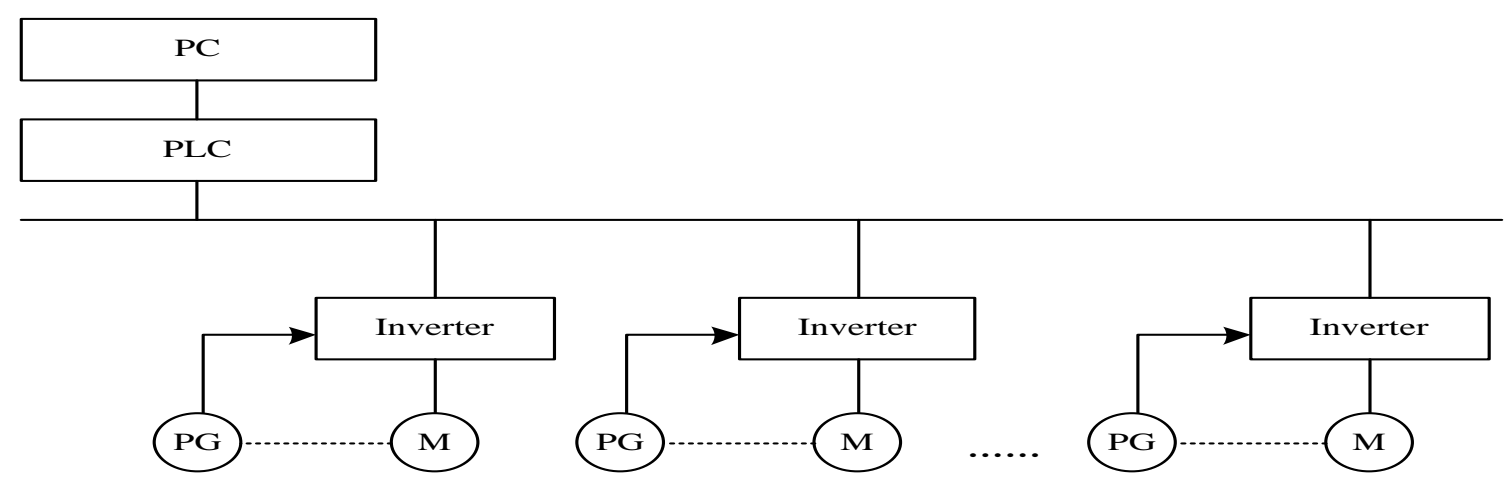

Fig. 2 Structure of automatic control system for paper machine 
The system is mainly composed of the upper computer PC, the main controller PLC, the multiple frequency converters and the motor. There are two layers of network, which are the monitoring level network and the field level network. The whole system structure is simple, and the level is clear. The system is easy to expand.

\subsection{Communication design of control system}

This system is a typical paper machine automatic control system, and the field bus control method is adopted. The network is divided into two levels, which are field equipment level network and workshop monitoring level network. The field equipment includes the distributed I/O, the actuator, the field sensor, and some switching equipment. The field device level network is used to connect the field equipment to realize the control of the field equipment and the interlocking between each other. In the paper machine control system, the PROFIBUSDP field bus is used to make up the field equipment level network.

The workshop monitoring level network has mainly completed the monitoring of the workshop equipment. The main equipment of the workshop is connected, and the monitoring is realized through the industrial control machine. Workshop monitoring mainly includes the on-line monitoring of production equipment, equipment fault monitoring and alarm, production statistics, production scheduling and so on. The industrial control machine of the workshop monitoring network is generally placed in the monitoring room, which is usually equipped with printing equipment and so on. For workshop monitoring, it is mainly the collection of some production data. Therefore, the speed of data transmission is not the most important, the amount of transmit data is important.

The system uses multi point interface (MPI) network for this level network. MPI communication is a simple and economical way of communication that can be used when the rate of communication is not high. The communication rate of MPI network is $19.2 \mathrm{kbit} / \mathrm{s}$ to $12 \mathrm{Mbit} / \mathrm{s}$ with a maximum connection of 32 nodes. The maximum communication distance is $50 \mathrm{~m}$. However, the communication distance can be extended by a repeater. When using the MPI network, a special communication network interface C05611 card needs to be installed on the host computer. The MPI network has a large amount of information transmission, which can meet the requirements of the system. PLC connects with the industrial control machine through the MPI network, and transfers the collected data to the PC computer. On the upper industrial computer, the corresponding configuration programs are compiled to display the relevant data in real time.

The field device level network mainly refers to the communication between the PLC and the frequency converter. The system uses the field PROFIBUS bus. PROFIBUS is an open field bus. The automation equipment of the manufacturer can be connected to the PROFIBUS bus for data exchange. The field device level network PROFIBUS bus in this system mainly connects SIEMENS's PLC, Emerson's inverter and distributed I/O. The transmission speed of PROFIBUS can be selected in the range of 9.6kbaud-12Mbaud. In addition, when the bus system is started, all the devices connected to the bus are set to the same communication rate.

PROFIBUS bus is widely used in the automation system of various industries. More common areas are: automation in manufacturing, automation in process industry, and automation in buildings, transportation and electricity, and so on.

PROFIBUS is a field bus technology that is used for factory automation, field device level and factory monitoring level data communication. It can realize decentralized digital control and field communication network from field device level to workshop monitoring. Its appearance provides a practical solution for the realization of the integrated automation of the factory and the intellectualization of the field equipment.

\subsection{Software design of control system}

Paper making process is a more complex process, including chemical, mechanical, electrical and other aspects of knowledge. Mainly for the automatic control system of paper machine is designed in this paper is made part of the papermaking process. The automatic control system of paper machine designed in this paper is mainly aimed at the paper making process. According to the above analysis, it is known that the paper making process is mainly realized by the synchronous operation of multiple speeds motor. In the whole system, the speed requirements of each electric motor are different. PLC controls the operation of the whole system, and the software is also written for PLC. According to the requirements of control, the program in PLC first checks whether the communication between the master station (i.e. PLC) and the EV3000 converter connected to the connection between the master and the slave station (TDS-PA01 field bus adapter) is normal or not. If it is found that the communication is not normal, it needs to be dealt with. For example, the communication is restored to normal by activating the way from the station. If the communication is normal, the PLC runs the data communication subroutine between the master station and the slave station, and carries out the related data exchange. Then, PLC will check whether it meets the starting condition of inverter or not. If it is satisfied, the frequency converter is started, and if it is not satisfied, it enters the waiting state and continues to check if the starting state of the inverter is satisfied. When the starting condition of the frequency converter is met, PLC will check if 
the frequency converter fails. If it fails, it will enter the alarm state and handle the fault. Until the frequency converter has no fault, the PLC can control the starting frequency converter at this time. After the frequency converter starts, the PLC will detect the external input signal to determine what speed to set for each inverter and whether there is speed regulation for each inverter. In addition, according to these data, the motor which is dragged by the inverter is variable frequency and speed regulating.

The effect of load distribution control based on speed control is relatively poor, while the load distribution based on torque control is more accurate. Therefore, the system adopts torque control mode load distribution. The specific software flow chart is shown in figure 3 . The $\mathrm{K}$ in the graph is a constant.

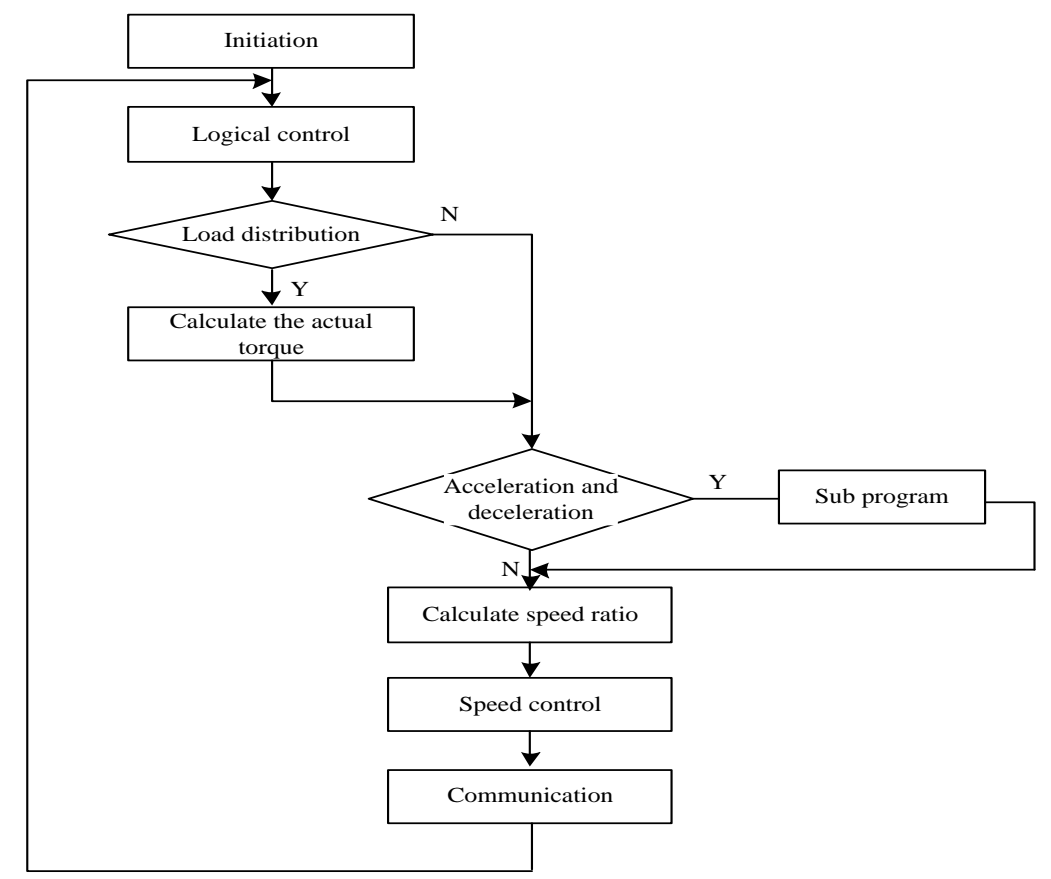

Fig. 3 The software flow chart of the paper machine automatic control system

\section{Simulation Study}

\subsection{Simulation of speed chain control}

The simulation structure of the speed chain control system will be simulated in three forms, the master- slave structure, the parallel structure and the coupling structure. Finally, the simulation results of various structures are compared. The simulation structure of the coupled synchronous control system is mainly controlled. The structure diagram is shown in figure 4 .

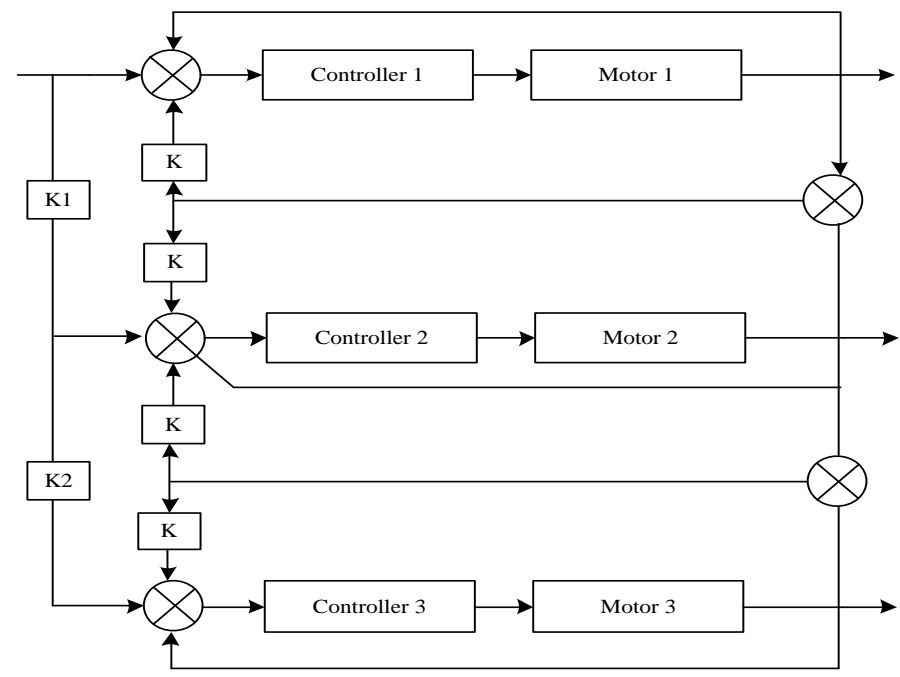

Fig. 4 Simulation structure of coupled synchronous control system 


\subsection{Research on the simulation of speed chain control system}

In the simulation study, the control object is the speed chain synchronization control system composed of 3 three-phase asynchronous motors controlled by vector. The speed is set to $300 \mathrm{r} / \mathrm{min}$. At $0.2 \mathrm{~s}$, the load suddenly changes from $0 \mathrm{Nm}$ to $12 \mathrm{Nm}$. The PI control algorithm and the auto disturbance rejection control algorithm are used in the simulation algorithm.

The parameter tuning of the PI control algorithm is made up of a trial and error method. The design and parameter tuning of the ADRC are described as follows: The first order ADRC is designed in the simulation process. The extended state observer (ESO) only outputs the observed values of the control object and the unknown disturbance term without the differential output term of the controlled object. Therefore, there is no the tracking differentiator (TD) in the design of the controller. The arrangement of the transition process is mainly aimed at the design of the servo system.

This system is the speed control of asynchronous motor, and it is not necessary to arrange the transition process. Therefore, for the controller studied in this paper, the TD link is not necessary. It can be omitted in the ADRC model.

Auto disturbance rejection control (ADRC) is also a control method based on error feedback, which has strong robustness. Its control performance is superior to the traditional control algorithm. However, there are many parameters involved in the design of ADRC algorithm. After determining the structure of the ADRC, the reasonable selection of the parameters will mainly affect the performance of the controller. The following is the principle and method of parameter selection of the first order ADRC.

The parameters of the ESO and the nonlinear state error feedback control law (NLSEF) should be adjusted. In addition, the tracking effect of ESO in real time is considered. In order to facilitate the implementation in the practical application, the parameter generally takes about 0.5 corresponding to the integer square algorithm.

There are two main reasons for this selection: First, the characteristics of nonlinear feedback are maintained. Without a large feedback gain, the rapid attenuation of the error can be achieved. Secondly, the computation of root operation is relatively small and it not occupies the system operation for a long time. Meanwhile, it is easy to implement.

Figure 5 is a speed chain control simulation based on the coupling structure of the PI controller.

Figure 6 is the synchronization performance of three control structure speed chains.

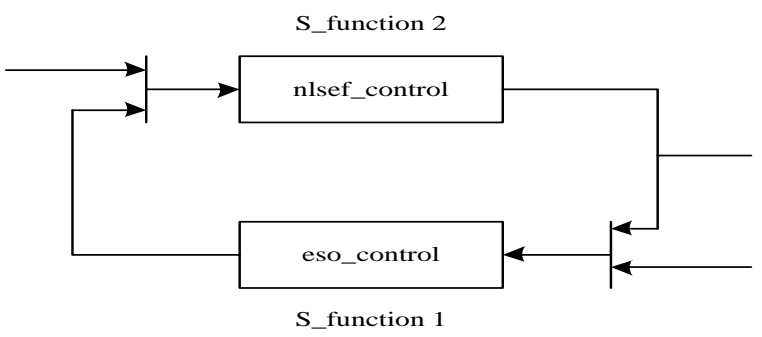

Fig. 5 The structure of auto disturbance rejection controller used in simulation research

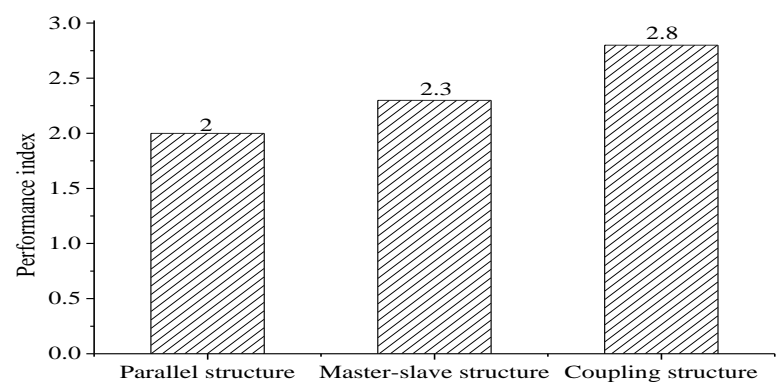

Fig. 6 Index comparison diagram of three kinds of control structures

\subsection{Simulation results discussion}

In the three control structures, the coupling structure > master-slave structure > parallel structure as far as the synchronization performance of the speed chain is concerned. The main reason is that there is a mutual influence between each motor. At the same time, the parallel structure is completely free from the mutual influence of the motor.

Therefore, its synchronization performance is the worst. However, for the coupling structure, there is a mutual influence between the two motors. Therefore, its synchronization performance is optimal. For the master-slave synchronization structure, although there are interconnected connections between the two motors, they are unidirectional. The change in the speed of the rear motor has no feedback on the front motor and can only affect the back motor. Therefore, its synchronization performance is between the other two motors. Considering the antiinterference ability of each motor to load disturbance, the speed chain synchronization system using ADRC is superior to the ordinary PI controller in overshoot, dynamic performance, steady state accuracy and anti-jamming performance. In addition, good control performance and reasonable control synchronization structure make the synchronization performance of the whole system more superior.

In this study, MATLAB software is used as a simulation tool to simulate the synchronous control of speed chain. Three control strategies are adopted, namely, parallel synchronization, master and slave synchronization and cross coupling synchronization. 
Moreover, the application effect of the traditional PI controller and the auto disturbance rejection controller in the three-phase asynchronous motor speed regulation is compared. Through the comparison of simulation waveforms, it is shown that in the three control strategies, the cross coupling synchronization of the speed chain synchronization is the best. The effect of the parallel synchronization is poor, and the effect of the master and slave synchronization is between the two strategies. Comparing the simulation waveforms on the control algorithm, it is known that the speed chain synchronization control effect using ADRC is better than the traditional PI controller in regulating time, overshoot and anti-disturbance performance.

\section{Conclusion}

For the paper machine control system, speed chain synchronization control, constant tension control and load distribution control are first analysed, and the control mechanism and control strategy are summarized. Then, taking MATLAB as a simulation tool, the simulation of speed chain control is achieved. The parallel synchronization, master-slave synchronization and cross coupling synchronization are carried out respectively.

Then, the theory of ADRC is introduced in detail, and it is applied to the simulation of speed chain synchronization control. Compared with the control effect of the traditional PI controller, it is concluded that the speed chain synchronization control system with active disturbance rejection controller is better than the ordinary PI controller in regulating time, overshoot and disturbance resistance. After finishing the theoretical analysis and simulation of the paper machine automatic control system, the actual situation of a paper industry is combined. Then, a set of automatic control system for paper machine based on PLC is designed. The system takes PLC as the main controller. The network structure is divided into two levels, which are field equipment level network and workshop monitoring level network.

\section{References}

[1] Maciejko W (2015). The Effect of Voice over IP Transmission Degradations on MAP-EM-GMM Speaker Verification Performance. Archives of Acoustics, 40(3), 407-417.
[2] Guangqing N (2017). Application of automatic control technology of drilling machine based on PLC. Acta Technica Csav, 2017, 62(1), 531-540.

[3] Narang I, Bharota I K, Raisuddin S, et al (2015). Design and Experimental Research of Automatic Control System of Material Transport Car Based on Siemens S7-300 PLC. Applied Mechanics \& Materials, 703(1), 277-280.

[4] Kaluz M, Garciazubia J, Fikar M, et al (2015). A Flexible and Configurable Architecture for Automatic Control Remote Laboratories. IEEE Transactions on Learning Technologies, 8(3), 299-310.

[5] Martinov G M, Nezhmetdinov R A, Kuliev A U (2016). Approach to implementing hardwareindependent automatic control systems of lathes and lathe-milling $\mathrm{CNC}$ machines. Russian Aeronautics, 59(2), 293-296.

[6] Wang D, Yi S, Yang Q (2017). Design of wood drying control system based on PLC. Acta Technica Csav, 2017, 62(2), 351-356.

[7] Yin Y, Yue Z (2016). Research and Design of Coal PLC Control System in Coal-fired Power Plants. International Journal of Smart Home, 10(1), 1-8.

[8] Hou X (2015). Electric control system of numerical control machine tool based on PLC. Acta Technica Csav, 2017, 62(2), 265-272.

[9] Cabral F G, Moreira M V, Diene O, et al (2015). A Petri Net Diagnoser for Discrete Event Systems Modeled by Finite State Automata[J]. IEEE Transactions on Automatic Control, 60(1), 59-71.

[10] Rodríguez F, Maria D M C, Sánchez J A, et al (2016). Architecture to develop semi-virtual industrial laboratories for the interactive learning of process automation. Computer Applications in Engineering Education, 24(3), 335-346.

[11] Yang G, Jiang T, Hou L, et al (2017). A Control System of PLC's Stereo Garage Based on Photoelectric Sensor. International Journal of Online Engineering, 13(7), 120.

[12] Cohen Y (2015). A technique for integrated modelling of manual and automatic assembly. Journal of Manufacturing Technology Management, 26(2), 164-181.

[13] Kennedy D (2015). Quantitative analysis of acid chlorides using automatic cold on-column injection. Journal of Separation Science, 11(4), 350-351. 